\title{
Hydra tropomyosin TROP1 is expressed in head-specific epithelial cells and is a major component of the cytoskeletal structure that anchors nematocytes
}

\author{
Maria S. López de Haro, Luis M. Salgado*, Charles N. David and Thomas C. G. Bosch ${ }^{\dagger}$ \\ University of Munich, Zoological Institute, Luisenstraße 14, 80333 Munich, FRG \\ *Present address: Instituto de Biotecnologia - UNAM, Departmento de Bioquimica, Apartado 510-3, 62271 Cuernavaca, México \\ †Author for correspondence
}

\section{SUMMARY}

A cDNA clone encoding a 253 amino acid tropomyosin was isolated from Hydra in a differential screen for headspecific genes. The Hydra tropomyosin gene, designated trop1, is a single copy gene, lacks introns and is strongly expressed in tentacle-specific epithelial cells. Analysis of protein synthesis in head and gastric tissue indicated a high rate of tropomyosin synthesis in head tissue. Immunolocalization of tropomyosin in tentacle tissue revealed a cushion-like tropomyosin-containing structure within battery cells at the base of nematocytes. The structure appears to form part of the cytoskeletal anchor for nematocytes. Tropomyosin cushions were also observed in epithelial cells along the body column, which contain mounted stenotele nematocytes.

Key words: coelenterate, cytoskeleton, $H y d r a$, nematocyte, tropomyosin

\section{INTRODUCTION}

Hydra tissue consists of two cell layers, ectoderm and endoderm. Both cell layers are composed of only a few cell types derived from two major pools: interstitial cells, which differentiate into nerve cells, nematocytes and gland cells, and epithelial cells. Epithelial tissue in Hydra is produced continuously causing displacement of epitheliomuscular cells toward the extremities. The continuous change of axial location in the animal is accompanied by changes in the epithelial cell phenotype. In the region beneath the head, termed the 'tentacle formation zone' (Hobmayer et al., 1991), ectodermal epitheliomuscular cells differentiate into tentacle epithelial cells, which then take up nematocytes to form a battery cell complex (Campbell, 1987). Nematocytes are anchored in battery cells by a specialized intercellular junction termed the nematocyte/battery cell/mesoglea complex (Wood and Novak, 1982). The complex consists of a tongue of the epithelial cell that extends between the base of the nematocyte and the underlying mesoglea. The epithelial tongue is connected to the nematocyte by a desmosome and to the underlying mesoglea by a hemidesmosome. The epithelial cell cytoplasm between the junctions contains microtubules and bundles of filaments that do not stain with phalloidin.

Little is known about the molecular events accompanying the differentiation of an epitheliomuscular cell into a tentacle cell. In a differential screening approach designed to isolate cDNA clones for head-specific epithelial cells we have isolated and characterized a gene with sequence homology to tropomyosin. A polyclonal antibody specific for tropomyosin reacted specifically with the nematocyte/battery cell/mesoglea complex, suggesting that tropomyosin is a major part of this complex.

Tropomyosins are a family of actin-binding cytoskeletal proteins that are found in all eukaryotic organisms (Lees-Miller and Helfman, 1991). Each tropomyosin molecule is a rodshaped coiled-coil dimer assembled from highly $\alpha$-helical monomers. Two groups of tropomyosin have been described, a low molecular mass form present in nonmuscle cells and a high molecular mass form present in muscle cells. In higher eukaryotes the diversity of tropomyosin isoforms is due to the presence of several tropomyosin genes, as well as to the alternative splicing of RNA transcripts encoded by these genes (for reviews see Lees-Miller and Helfman, 1991; Gerisch et al., 1991).

\section{MATERIALS AND METHODS}

\section{Hydra strains, culturing and radiolabeling}

Polyps of Hydra magnipapillata (strain sf-1) and Hydra vulgaris were cultured using standard procedures. To prepare Hydra tissue consisting of epithelial cells only, polyps of $H$. magnipapillata strain sf- 1 , a temperature-sensitive mutant, were cultured at $28^{\circ} \mathrm{C}$ for 4 weeks (Marcum et al., 1980). To detect newly synthesized proteins, polyps were radiolabeled by injecting $25 \mu \mathrm{Ci}$ of ${ }^{35} \mathrm{~S}$-labeled amino acids (Trans $\left[{ }^{35} \mathrm{~S}\right] \mathrm{label}$, ICN Radiochemicals, $1100 \mathrm{Ci} / \mathrm{mmol}$ ) into the gastric cavity as described (Bosch et al., 1988).

cDNA libraries and differential screening

To construct an epithelial cell-specific cDNA library, Hydra tissue 
consisting of epithelial cells only was prepared by culturing $H$. magnipapillata strain $\mathrm{sf}-1$ at $28^{\circ} \mathrm{C}$ for 4 weeks. Poly(A) ${ }^{+}$from epithelial polyps was used to construct a cDNA library in $\lambda$ ZAPII (Stratagene). The library was screened with ${ }^{32} \mathrm{P}-$ labeled cDNA made from gastric and head tissue of epithelial polyps; 19 recombinant phage were detected by the head-specific cDNa probe and 17 of these clones encoded the same gene. The remaining 2 clones were derived from different genes. One of them, initially designated $k s-3$, is described here. A second $\lambda$ ZAPII cDNA library was prepared commercially (Stratagene) from Hydra vulgaris whole polyp poly $(\mathrm{A})^{+}$RNA and was generously provided by Drs Michael Sarras (University of Kansas Medical Center) and Hans Bode (University of California, Irvine).

\section{Molecular techniques}

Sequence analysis, nucleic acid isolation, DNA and RNA blot analysis were carried out following standard procedures (Sambrook et al., 1989). For in vitro translation, a plasmid with a complete coding sequence for the TROP1 protein was transcribed with T7 RNA polymerase. In vitro translation of the purified RNA was carried out in a nuclease-treated rabbit reticulocyte lysate (Ambion) in the presence of $\left.{ }^{35} \mathrm{~S}\right]$ methionine. In situ hybridization on whole mounts and macerates was carried out as described (Kurz et al., 1991). All polymerase chain reactions (PCR) were carried out using the buffer conditions and $T a q$ polymerase supplied by the manufacturer (Amersham). For reverse transcriptase-PCR (RT-PCR) total RNA was prepared from polyps as described and $1 \mu \mathrm{g}$ was used to prepare oligo(dT)-primed cDNA. One-tenth of the reaction was used as template for 30 cycles of PCR with different pairs of primers. Oligonucleotides were synthesized on a Pharmacia LKB synthesizer. DNA sequences were analysed using the Hibio DNASIS/PROSIS program (Hitachi). EMBL Database accession number for tropl, X70840.

\section{Antibodies and immunoblotting}

Polyclonal anti-chicken tropomyosin antibody was purchased from Sigma Chemie. The anti-yeast tropomyosin antibody was a gift from A. Bretscher, Ithaca. Total Hydra protein was analyzed as described (Bosch et al., 1989). For western blot analysis, the secondary antibody was coupled to horseradish peroxidase. The filters were developed using the ECL chemiluminescence system (Amersham Corp.).

\section{Immunofluorescence microscopy}

H. magnipapillata strain sf-1 polyps were anesthetized in $2 \%$ urethane and fixed with cold $\left(-20^{\circ} \mathrm{C}\right)$ methanol for 15 minutes. The polyps were then washed in PBS and incubated in blocking solution (PBS, $1 \%$ BSA) for 30 minutes. Anti-chicken tropomyosin antibody was used at a dilution of 1:200 in blocking solution. The polyps were exposed to the primary antibody for 1-2 hours. They were washed with PBS and incubated with rhodamine isothiocyanate (TRITC)labeled mouse anti-rabbit IgG (Pierce Chemical Co.) at a dilution 1:50 for 1 hour. Tropomyosin from chicken muscle ( $1 \mathrm{mg} / \mathrm{ml}$, Sigma) was used to neutralize the tropomyosin antibody. To visualize actin filaments, animals were stained for 15 minutes with TRITC-phalloidin (Sigma, $0.1 \mu \mathrm{g} / \mathrm{ml}$ ).

\section{RESULTS}

\section{cDNA cloning and sequence analysis of the Hydra tropomyosin gene, trop1}

An epithelial cell cDNA library was constructed and differentially screened with labeled cDNA from head and body column tissue. One of the head-specific clones, initially named $k s-3$, had a 222 bp insert. To complete the sequence, a second cDNA library (gift from M. Sarras and H. Bode, see Materials and
Methods) was screened with the ks-3 insert and two clones were isolated. Restriction maps of the two clones indicated that both clones were derived from the same gene. A restriction map of clone ks-3-1 is shown in Fig. 1.

The $1.0 \mathrm{~kb}$ insert of $k s-3-1$ corresponded in size to the $1 \mathrm{~kb}$ transcript on northern blots (see Fig. 7). Sequence analysis indicated the presence of a single open reading frame of 253 amino acids beginning at nucleotide 102 (Fig. 2). The $5^{\prime}$-most ATG triplet is preceded by an A residue 3 nucleotides upstream and is immediately followed by a $\mathrm{G}$, which conforms to the Kozak consensus sequence of eukaryotic translation initiation sites (Kozak, 1987). The predicted protein product has a molecular mass of 29,216 Da.

A comparison of the predicted amino acid sequence of $k s$ 3-1 with the Swiss protein data base revealed a high degree of similarity to tropomyosins (Fig. 3). We, therefore, have named this gene tropl, for tropomyosin gene 1. A characteristic feature of tropomyosin is a repeating pattern of non-polar and polar residues (Smilie, 1979). As shown in Fig. 4, this repeating pseudoheptapeptide pattern, which is responsible for the stabilization of the coiled-coil structure, is also found in Hydra TROP1. As with all tropomyosins sequenced so far, TROP1 is devoid of the helix-breaking residue proline, suggesting a secondary structure with high $\alpha$-helical content. Detailed comparison of the TROP1 sequence with various muscle and non-muscle tropomyosins indicated that Hydra TROP1 does not easily fit in either category (see Discussion).

\section{Hydra trop1 is a single copy gene lacking introns}

To estimate the number of genes encoding tropomyosin, restriction digests of total Hydra genomic DNA were probed with either the 5' 280 bp EcoRI fragment or the 3' PstI/EcoRI fragment of clone ks-3-1 (see Fig. 1). Fig. 5 shows that single bands of hybridization were found when the genomic DNA was cut with enzymes that have no restriction site in the cDNA probes. In lane 4 the weakly hybridizing band of $>12 \mathrm{~kb}$ appears to be due to partial PstI digest. The pattern of hybridization was similar after washing under low and high stringency. Thus, there appears to be only one tropomyosin gene in Hydra.

In organisms as diverse as Drosophila and man, tropomyosin genes give rise to a multiplicity of isoforms due to the presence of multiple introns and alternate splicing (for Drosophila, see Karlik and Freyberg, 1986; for man, see Wieczorek et al., 1988). Thus, we examined the Hydra tropomyosin gene for the presence of introns using the polymerase chain reaction (PCR). PCR was carried out using genomic DNA. Fig. 6A indicates that the primers flank a region, which, e.g. in rat tropomyosin, is interrupted by several

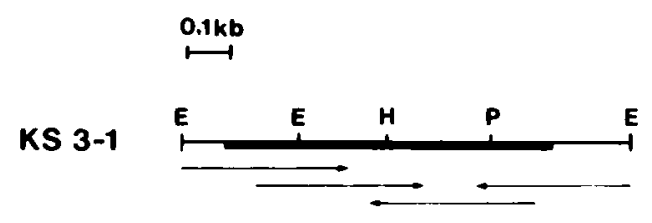

Fig. 1. Restriction map of cDNA clone $k s-3-1$. The coding sequence is represented by a thick line. Arrows show the strategy used for sequence analysis. Restrictions sites: EcoRI (E), HindIII (H), PstI (P). 
introns of variable length (Ruiz-Opazo and Nadal-Ginard, 1987). Thus, if Hydra tropl contains introns, one would expect to find PCR amplification products larger than those obtained with mRNA (cDNA) as template. Fig. 6B shows that for four primer pairs the size of the amplified fragment was that predicted from the cDNA sequence. In addition, using primer pair $1 / \mathrm{C}$ the identical fragment was amplified when using cDNA from either head or gastric tissue (Fig. 6B, lane 1 and 2), or genomic DNA (Fig. 6B, lane 3). Control PCR reaction devoid of genomic DNA did not give any signal (data not shown). Southern analysis of amplified DNA using radiolabeled $k s-3-1$ as probe revealed that the intense 700-900 bp bands observed in Fig. 6B hybridize to tropl (data not shown). The results, which are consistent with the Southern hybridization pattern described above (Fig. 5), indicate that in contrast to higher metazoa (Ruiz-Opazo and Nadal-Ginard, 1987) but similar to yeast (Liu and Bretscher, 1989), the Hydra tropomyosin gene tropl lacks introns.

\section{Expression pattern of Hydra trop1}

To examine the level of tropl transcripts in cells from head and gastric tissue, we used the 3'PstI/EcoRI fragment of clone $k s-3-1$ (see Fig. 1) to probe a northern blot. An abundant $1 \mathrm{~kb}$ transcript was detected in RNA from head-specific cells (Fig.
7 , lane 1). In cells from gastric tissue (lane 2) only a low level of this transcript could be detected. The same observation was made when using RNA from epithelial polyps (data not shown), indicating that tropl is expressed at a high rate in head-specific epithelial cells.

In situ hybridization was carried out on macerates and whole mounts using the digoxigenin method (see Materials and Methods). As probe we used the $3^{\prime} P s t \mathrm{I} / E c o$ RI fragment of clone $k s-3-1$. Fig. 8 shows that tropl transcripts are abundant in tentacle-specific epithelial cells but undetectable in other cells (Fig. 8A). In whole mounts (Fig. 8B) the tentacles are clearly stained; body column tissue is unstained.

\section{Tropomyosin protein in Hydra}

A polyclonal antibody directed against tropomyosin and known to react with tropomyosins in a wide variety of species was used to determine the location of tropomyosin in Hydra battery cells. In western blots the antibody reacted specifically with a protein of apparent molecular mass $35 \mathrm{kDa}$ (Fig. 9A). Control preparations with the secondary antibody alone were unstained. To confirm that the $35 \mathrm{kDa}$ protein corresponds to the tropl gene product, synthetic tropl RNA was translated in vitro. In vitro translation of the synthetic RNA yielded a protein of $35 \mathrm{kDa}$ (Fig. 9B).

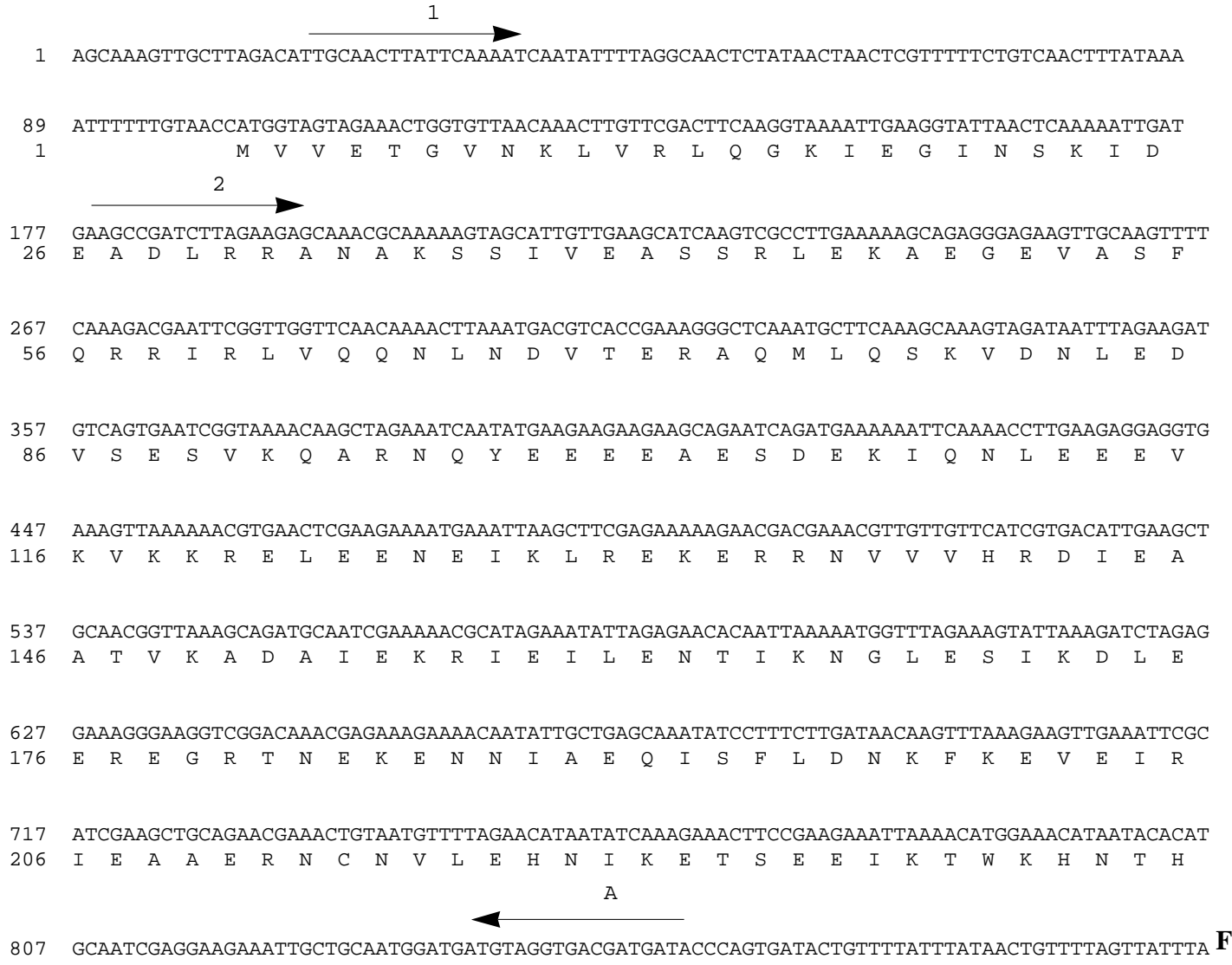

807 236 $\begin{array}{llllllllllllllllllllllllll}\text { A } & \text { I } & \text { E } & \text { E } & \text { E } & \text { I } & \text { A } & \text { A } & \text { M } & \text { D } & \text { D } & \text { V } & \text { G } & \text { D } & \text { D } & \text { D } & \text { T } & Q & * & *\end{array}$

TTGTTATTTATTTTAAATCTTTAATTGTTATTTTAGTTATTAGAATTTTTTTGAATAATAACAAAACACTTTATTCAAAAAATAACAAAA
ATGTATCAAACATTTTTGCCGATAAACTTGAATATCAAAACCTAAA 1030
Fig. 2. Nucleotide and deduced amino acid sequence of Hydra tropl cDNA. The stop codon is marked by asterisks. The overlined nucleotides mark the location of the synthetic oligonucleotides used for PCR (see Fig. 6). 
Fly ThI

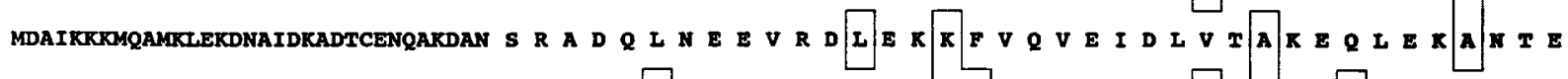
HumFib

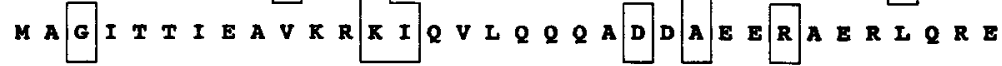

Yeast TPM1
40
50
60
70
80
90

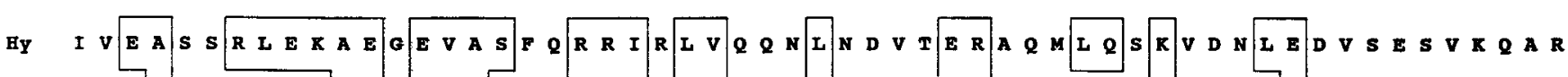

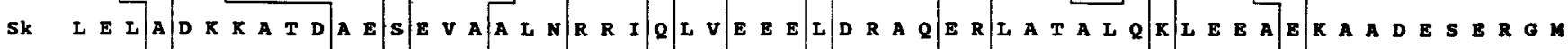

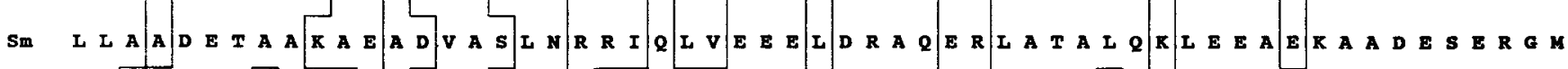

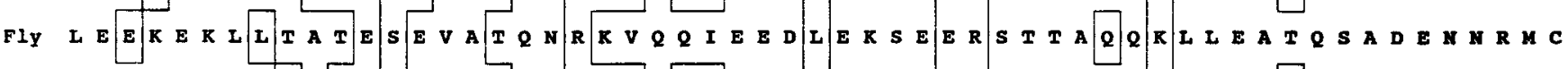

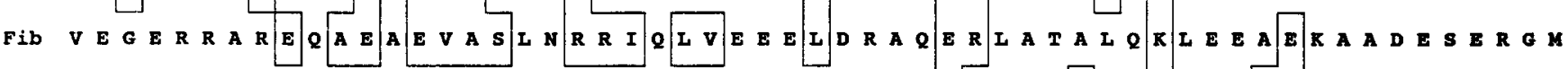
Yea MDKIREKLSNIKLEAESWQEKTEELRERDKDLEQEDVEKENQI

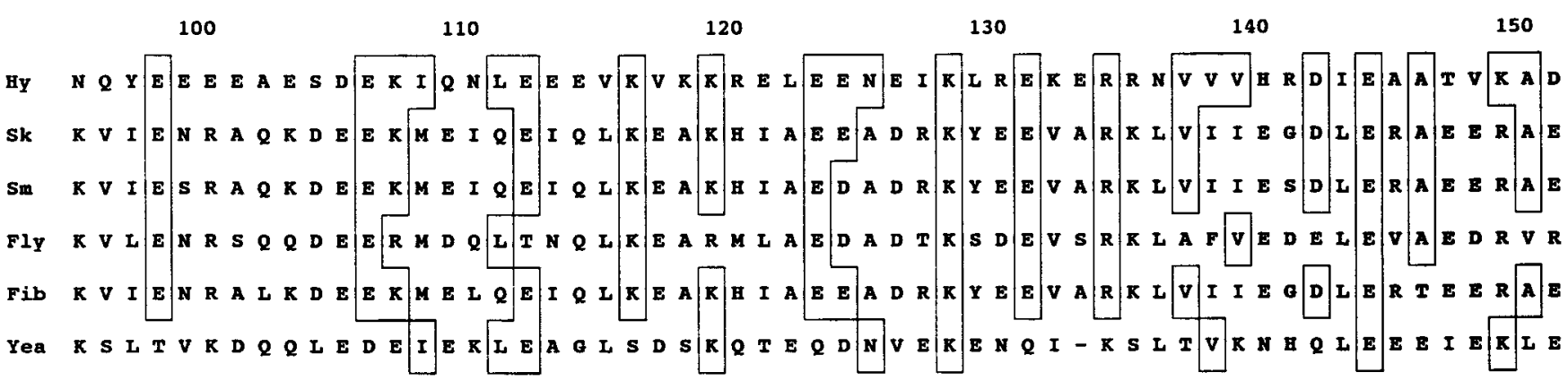
$160 \quad 170 \quad 180 \quad 190 \quad 200$

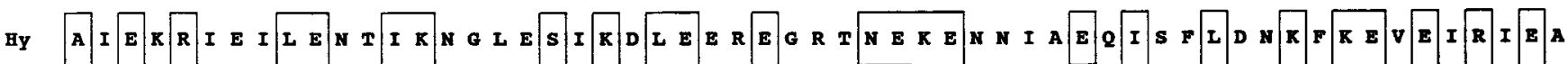

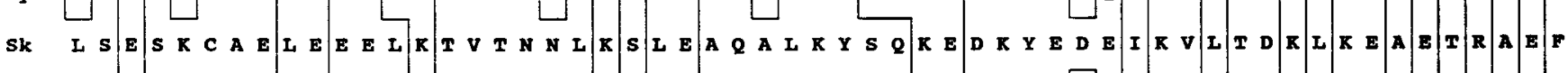

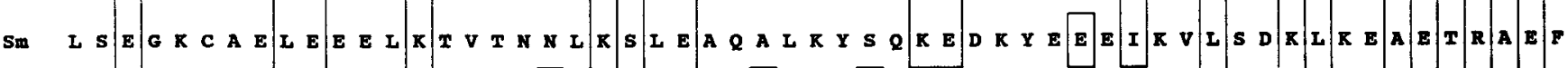

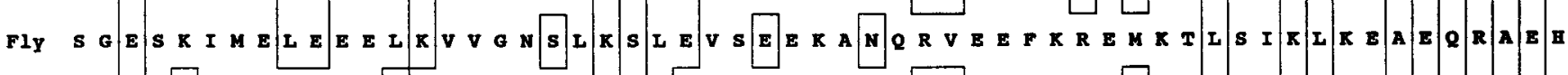

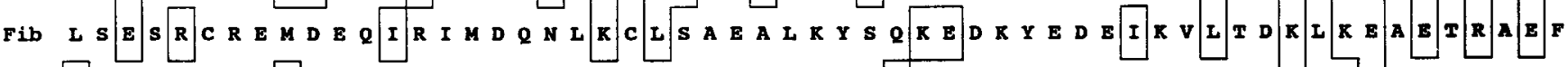

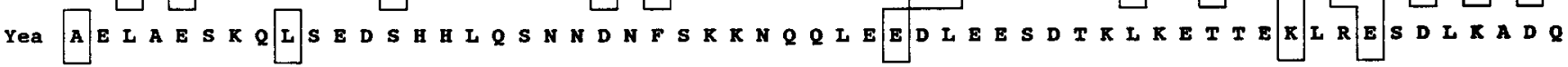

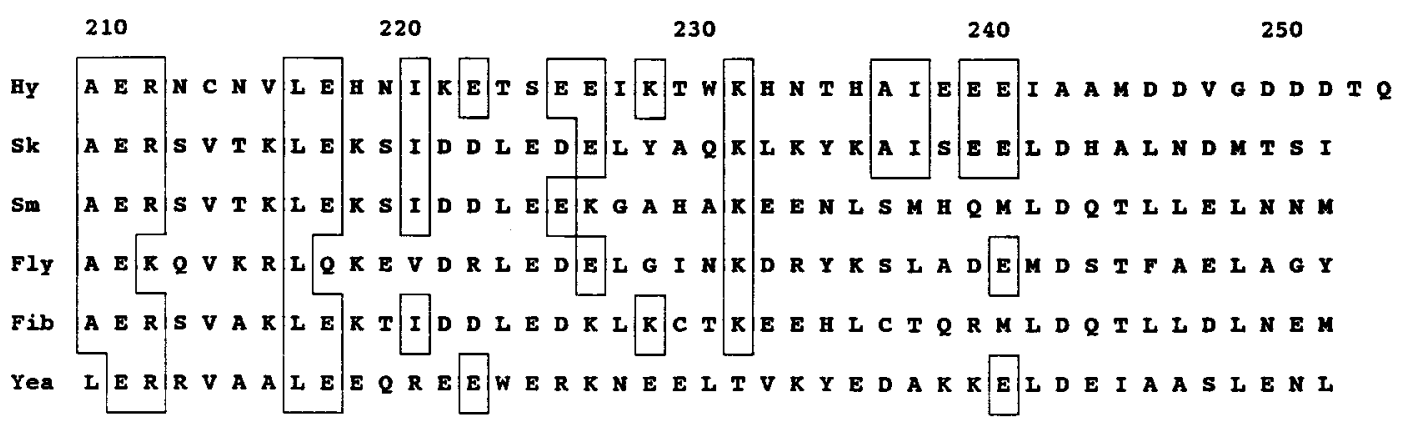

(253)

Fig. 3. Comparison of the amino acid sequence of Hydra TROP1 with tropomyosin sequences from various organisms. Identical amino acid residues are boxed. The amino acid sequences of the various proteins were obtained from the GenBank/EMBL Data Bank. Shown are sequences of skeletal muscle tropomyosin from quail (QuailSkel), smooth muscle tropmyosin from rat (RatSm) and Drosophila melanogaster (Fly TmI), and non-muscle tropomyosin from human (HumFib) and from Saccharomyces cerevisiae (Yeast TPM1). 


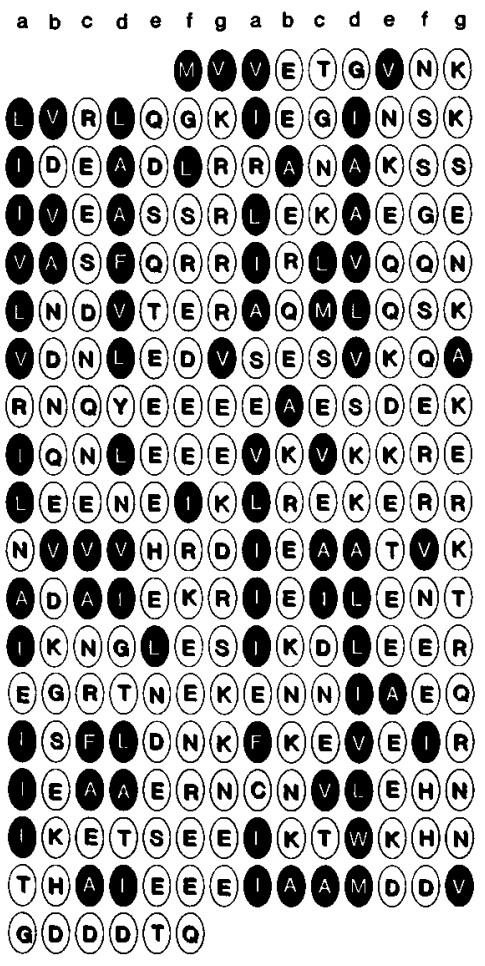

Fig. 4. Repeating pseudoheptapeptide pattern of non-polar and polar residues of Hydra TROP1. Non-polar residues are indicated by filled ellipses.

Since the northern analysis indicated increased levels of tropomyosin transcripts in head tissue, we looked for evidence of increased rate of tropomyosin synthesis in head tissue by labeling polyps with $\left.{ }^{35} \mathrm{~S}\right]$ methionine. Homogenates were prepared from head and gastric tissue and analysed by SDSPAGE (Fig. 9C). The pattern of newly synthesized proteins (Fig. 9C, panel 2) is different in head $(\mathrm{H})$ and gastric $(\mathrm{G})$ tissue.

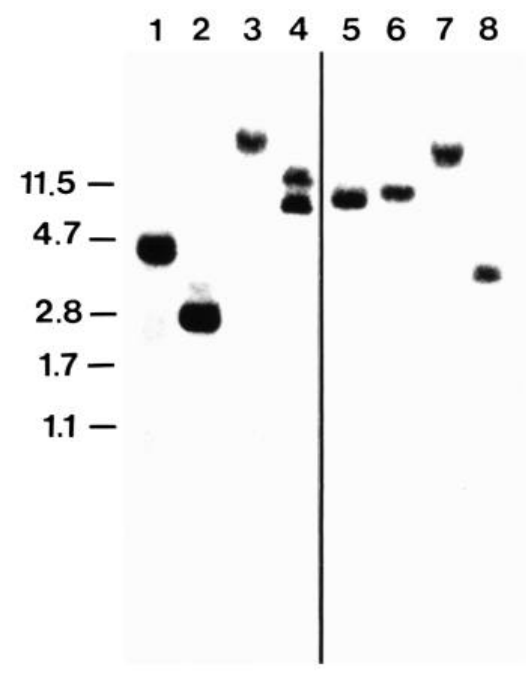

Fig. 5. Genomic Southern blot analysis of trop1. H. vulgaris DNA $(15 \mu \mathrm{g})$ was digested with EcoRI (lanes 1 and 5), HindIII (lanes 2 and 6), BamHI (lanes 3 and 7) and PstI (lanes 4 and 8),

electrophoresed on a $1.2 \%$ agarose gel, hybridized to either the $5^{\prime}$ $280 \mathrm{bp}$ EcoRI fragment of $k s-3-1$ (lanes 1-4) or the 3'PstI/EcoRI fragment of $k s-3-1$ (lanes 5-8) and washed at high stringency. No additional bands appeared when blots were washed at low stringency.

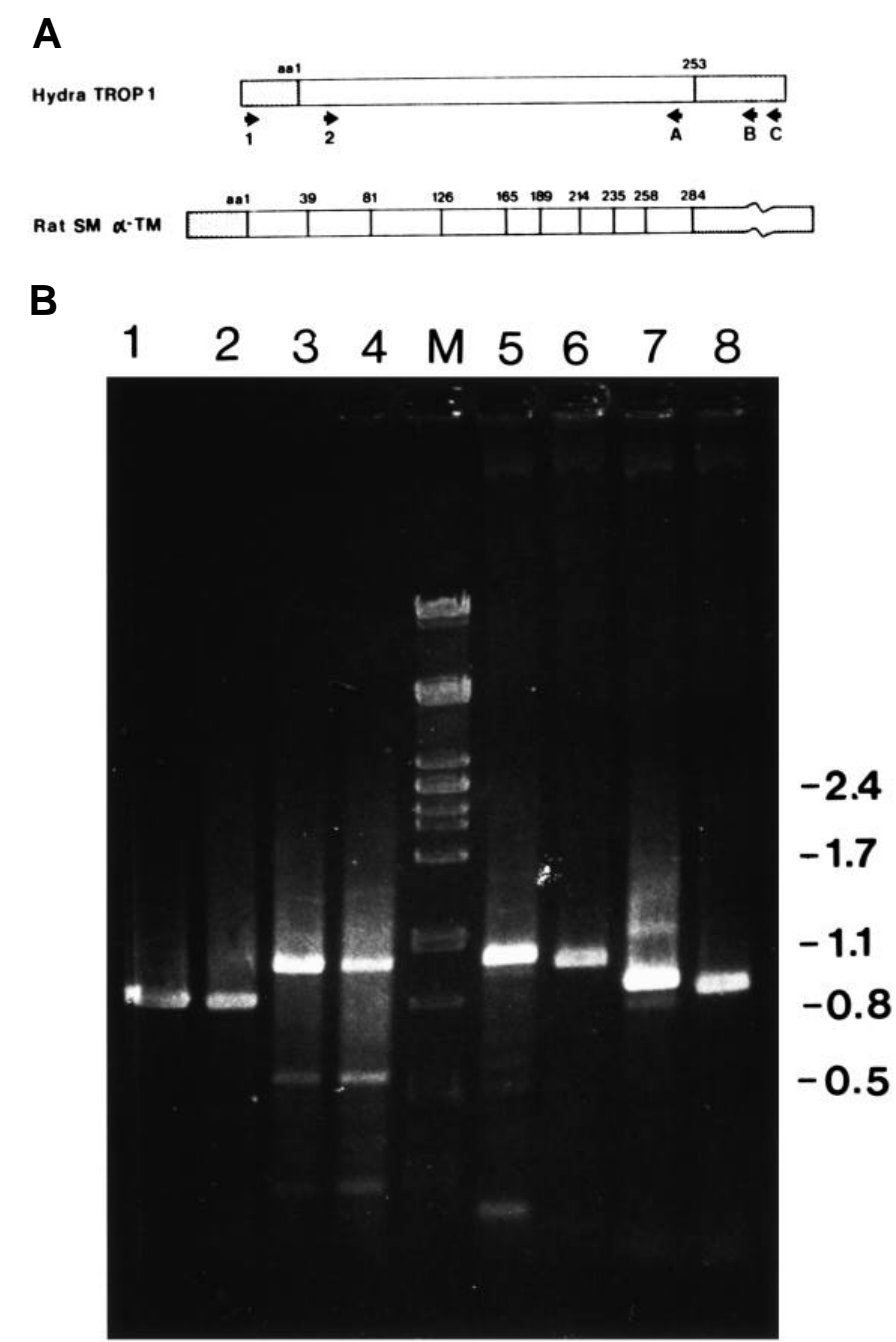

Fig. 6. PCR amplification of Hydra vulgaris cDNA and genomic DNA with tropomyosin-specific primers. (A) Position of primers in tropl sequence. For comparison, the exon organization of rat smooth muscle tropomyosin gene is drawn to scale. (B) PCR-amplified cDNA and genomic DNA. Lanes 1 and 2, RT-PCR using RNA from head (lane 1) and gastric tissue (lane 2) and primer set 1/C. Lanes 3 to 6 , PCR with genomic DNA. Lane 3, primer set $1 / \mathrm{C}$. Lane 4 , primer set 1/B. Lane 5, primer set 2/C. Lane 6, primer set 2/B. M, DNA size markers indicated in kilobases on the right.

In gastric homogenate the most prominent $\left[{ }^{35} \mathrm{~S}\right]$ methioninelabeled protein is of apparent molecular mass $45 \mathrm{kDa}$ and presumably represents Hydra actin. In the head homogenate several proteins are synthesized that are not as evident in gastric tissue. Among them is a protein of apparent molecular mass $35 \mathrm{kDa}$. Fig. 9C (panel 1) shows that this band reacts specifically with the tropomyosin antibody. Thus, there appears to be an increased rate of tropomyosin synthesis in head tissue concomitant with the differentiation of headspecific cells.

\section{Tropomyosin localization in Hydra cells}

To localize tropomyosin in Hydra cells, polyps were fixed in methanol at $-20^{\circ} \mathrm{C}$, stained as whole mounts with anti- 
12

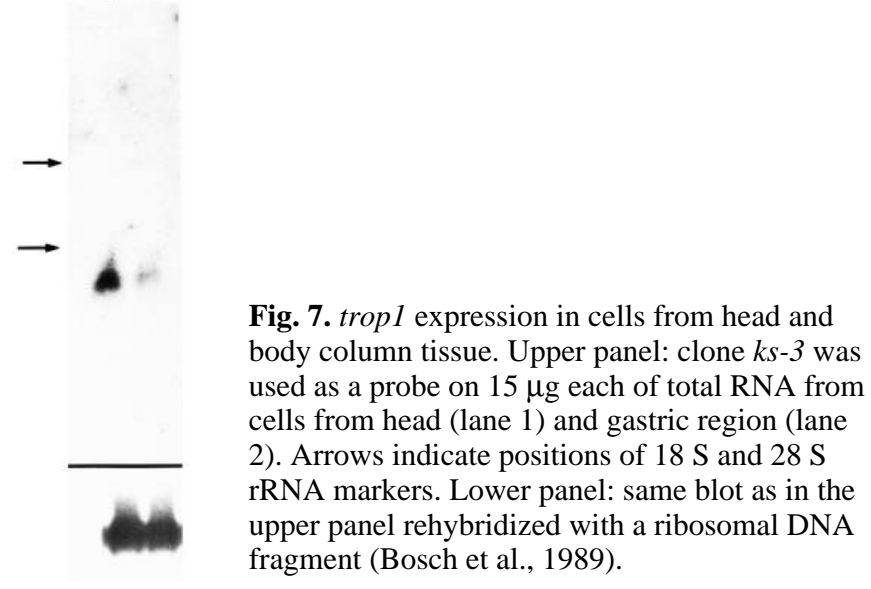

tropomyosin antibody and analyzed by epifluorescence microscopy and confocal scanning microscopy. In epithelial cells from the gastric region (Fig. 10A,B) diffuse tropomyosin staining was observed in the cytoplasm surrounding the large vacuoles of epithelial cells. This staining pattern appears to be similar to previous descriptions of cytoplasmic staining for tropomyosin in various cultured cell types. Localized tropomyosin staining was also observed along the muscle fibres in properly focused preparations.

In tentacle epithelial cells immunofluorescence staining for tropomyosin revealed a brightly stained condensed structure at the base of nematocytes (Fig. 10C,D). In favorable images this structure appeared to be closely adjacent to the mesoglea (Fig. $10 \mathrm{E}, \mathrm{F})$. The same result has been obtained with an antibody directed against yeast tropomyosin (data not shown). Control

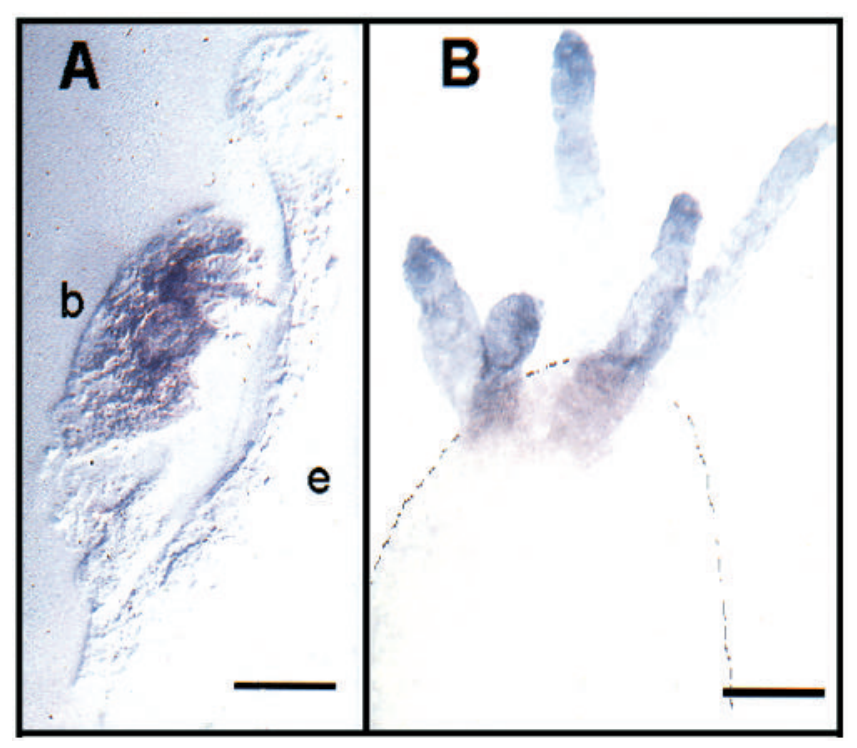

Fig. 8. In situ localization of tropl expression in macerated Hydra cells (A) and whole mounts (B). Polyps and cells were fixed and hybridized with the DIG-labeled 3' PstI/EcoRI fragment of clone $k s 3-1$ as described in Materials and Methods. Hybridization is restricted to tentacle-specific epithelial cells. b, battery cell; e, epithelial cell. Bars: (A) $10 \mu \mathrm{m}$; (B) $200 \mu \mathrm{m}$.

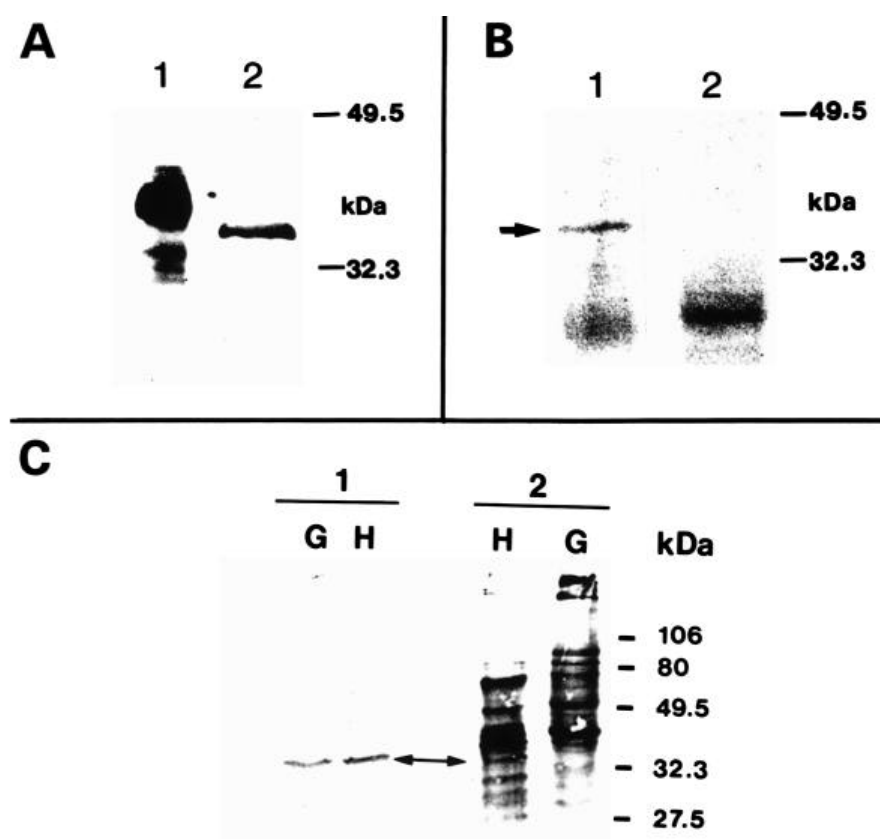

Fig. 9. Tropomyosin protein in Hydra. (A) Total muscle protein from dog (lane 1) and Hydra polyps (lane 2) probed with the polyclonal chicken tropomyosin antibody described in Materials and Methods. Note the different size of tropomyosin in Hydra and dog muscle. (B) In vitro translation products of trop1 RNA. Lane 1, product obtained from reticulocyte lysate when in vitro synthesized trop1 RNA was added to the lysate. Lane 2, results obtained from lysate to which no RNA was added. Arrow indicates position of trop 1 RNA translation product. (C) Immunoblot analysis of tropomyosin (panel 1) and pattern of newly synthesized proteins (panel 2) in gastric and head tissue of $H$. magnipapillata. $\left[{ }^{35} \mathrm{~S}\right]$ methionine-labeled protein from gastric $(\mathrm{G})$ and head tissue $(\mathrm{H})$ was first analyzed by immunoblotting using the tropomyosin antibody described in Materials and Methods (panel 1). Panel 2 shows the same filter after autoradiography indicating the pattern of newly synthesized proteins. The arrow points to a $35 \mathrm{kDa}$ protein that is detected by the tropomyosin antibody and is synthesized predominantly in the head region. Numbers shown at the right indicate positions of molecular mass markers.

preparations with secondary antibody alone did not give any specific fluorescence (data not shown). Preincubation of antibody with tropomyosin removed all binding activity (Fig. $10 \mathrm{I}, \mathrm{K})$.

Thin optical sections of tentacle epithelial cells were viewed by confocal laser scanning microscopy. As seen in Fig. 11B, the condensed tropomyosin staining appears as a cushion between the base of the nematocyst and the mesoglea. All four types of Hydra nematocytes, when embedded in battery cells, were found to be associated with this cushion-like tropomyosin structure. Large stenotele nematocytes had large cushions; small desmoneme nematocytes had small cushions. Epithelial cells containing mounted nematocytes in the body column also have a tropomyosin cushion at the base of the nematocyte. The cushion-like tropomyosin staining could not be correlated with any structure visible in phase-contrast or interference optics. Ultrastructurally, however, a tandem arrangement of desmosomes and hemidesmosomes between the base of the nematocyte and the mesoglea corresponds in location and shape to the 
tropomyosin staining shown in Fig. 11B. Wood and Novak (1982) have termed this junctional complex at the base of nematocytes the nematocyte/battery cell/mesoglea complex
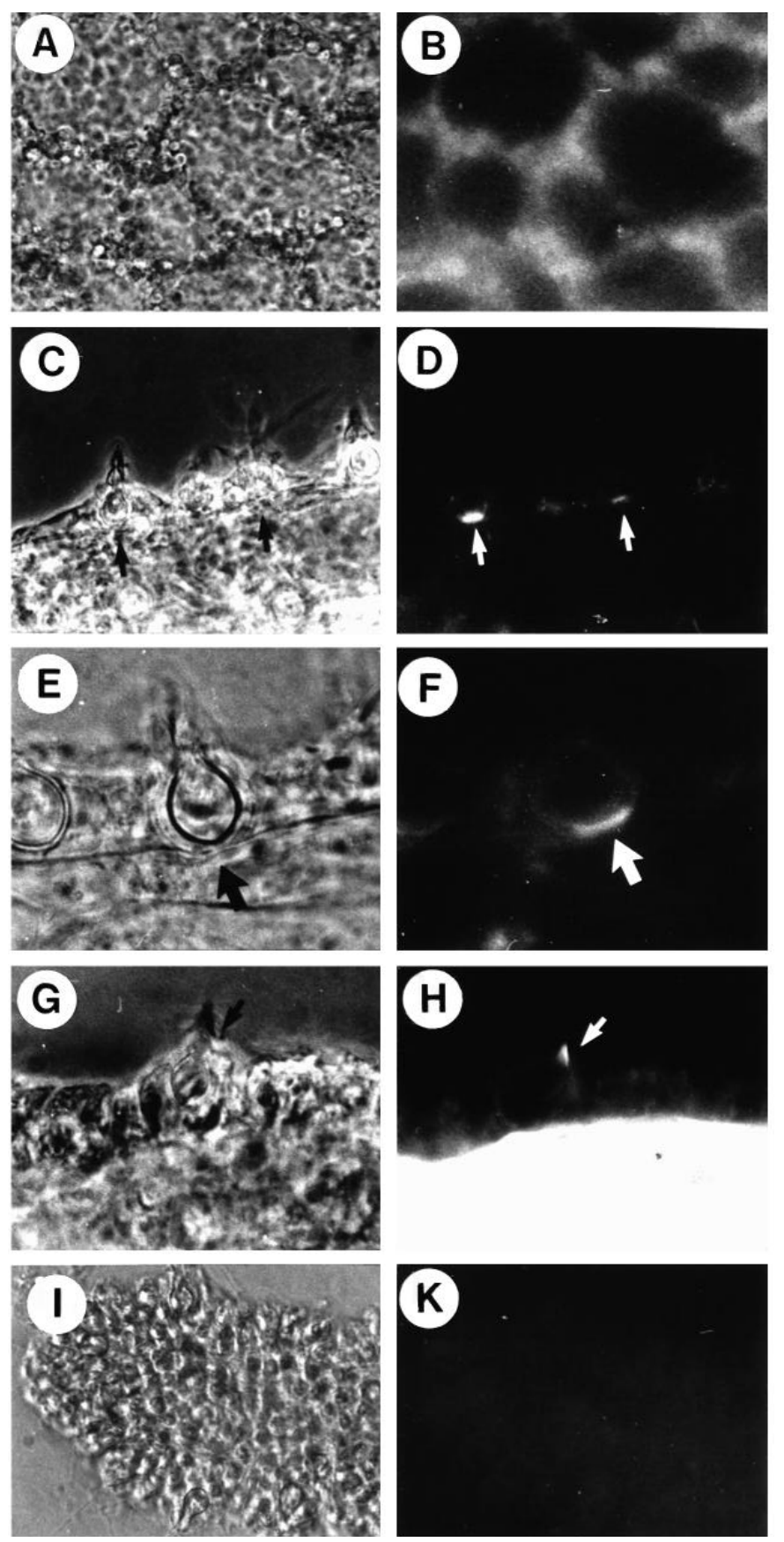

Fig. 10. Immunolocalization of tropomyosin in Hydra. $H$. magnipapillata polyps were fixed in cold methanol as described in Materials and Methods and incubated in tropomyosin antibody. Cells were visualized using phase-contrast (left-hand column) and fluorescence optics (right-hand column). (A,B) Gastric epithelial cells. (C,D) Tentacle epithelial cells. Note the condensed tropomyosin staining at the base of nematocytes (arrows).

(E,F) Higher magnification view of nematocyte mounted in tentacle epithelial cell. $(\mathrm{G}, \mathrm{H})$ Tentacle stained for actin with TRITCphalloidin. The arrow points to the cnidocil-supporting apparatus. $(\mathrm{I}, \mathrm{K})$ Tentacle stained with tropomyosin antibody, which was neutralized with tropomyosin as described in Material and Methods. and proposed that it functions as a nematocyst anchorage device.

To investigate whether actin is also present in the tropomyosin cushion, we stained whole mounts with TRITCphalloidin. F-actin staining was observed in longitudinal bands along the mesoglea corresponding to myonemes of epitheliomuscular cells (Fig. 10G,H). Staining was also detected in the cnidocil-supporting apparatus (arrow in Fig. 10H). We observed no phalloidin staining in the position of the tropomyosin cushion in agreement with previous observations (Wood and Novak, 1982; González Agosti and Stidwill, 1992)

To determine whether the condensed tropomyosin staining could also be observed in the absence of nematocytes, we stained a mutant strain depleted of interstitial cells and nematocytes by temperature treatment (see Materials and Methods). In Fig. 11C,D a partially depleted tentacle is shown with nematocytes present towards the tentacle tip but absent in the more proximal parts. No condensed tropomyosin immunoreactivity could be observed in areas of the tentacle that did not contain nematocytes. Thus, the observed tropomyosin structure at the nematocyte/battery cell/mesoglea junction is correlated with the presence of nematocytes.

\section{DISCUSSION}

We have cloned a cDNA that encodes a Hydra tropomyosin homolog. The full-length cDNA sequence (1030 bp) corresponds with the observed size of the tropl transcript $(1 \mathrm{~kb})$, indicating that the complete mRNA sequence has been obtained in cDNA form. The analysis of its primary structure has provided a number of interesting insights into the tropomyosin gene organization in Hydra and its relationship to other tropomyosins.

\section{TROP1 is member of a structurally distinct class of tropomyosins}

Two tropomyosin families (muscle tropomyosin and nonmuscle tropomyosin) have been described (Lees-Miller and Helfman, 1991). Hydra TROP1 is distinct from both of them in several features. (1) The Hydra TROP1 sequence is shorter than skeletal muscle tropomyosin (253 versus 284 amino acids) due to lack of 33 amino acids at the $\mathrm{N}$ terminus. (2) Several sites known in skeletal muscle tropomyosin to be of importance for binding troponin (Tyr261 and 267, cys190) are missing in TROP1. Furthermore, serine 283, which is phosphorylated in skeletal tropomyosin, is replaced by asparagine in Hydra TROP1. (3) The COOH-terminal portion of non-muscle tropomyosins is highly homologous, differing in only 6 of 23 positions in a variety of vertebrates; it is strikingly different from skeletal muscle tropomyosins. The carboxy terminus of Hydra TROP1 does not resemble the conserved non-muscle tropomyosin sequence; it is also different from yeast tropomyosin. Thus, Hydra TROP1 appears to be a structurally distinct member of the tropomyosin group of proteins.

\section{The Hydra trop1 gene has a very simple organization}

In higher eukaryotes the diversity of tropomyosin isoforms is due to the presence of several tropomyosin genes, as well as 

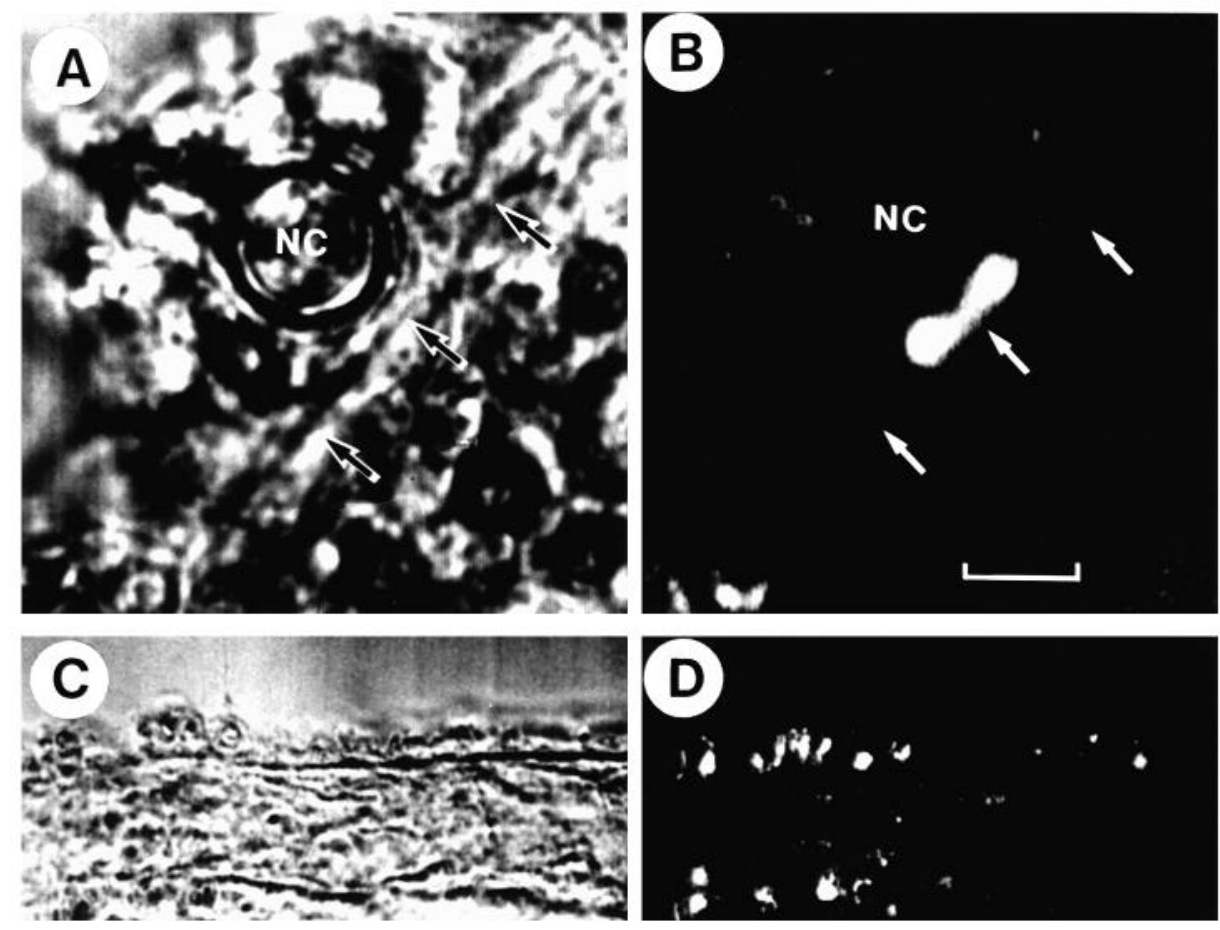

Fig. 11. (A, B) Confocal laser micrographs of a tentacle epithelial cell in the plane of a stenotele nematocyte stained with antitropomyosin (500 $\mathrm{nm}$ optical sections). Note the cushion-like structure at the base of the nematocyte. Bar, $5 \mu \mathrm{m}$. (C,D) Confocal laser micrograph of a tentacle partially depleted of nematocytes and stained with antitropomyosin antibody. Note that the staining is restricted to the nematocyte containing part (left-hand side of micrograph) of the tentacle. to the alternative splicing of RNA transcripts encoded by these genes (for review see Lees-Miller and Helfman, 1991). In contrast, hybridization analysis suggests that tropl in Hydra is likely to be the only gene of its type (Fig. 5). Furthermore, the single tropomyosin gene in Hydra lacks introns in regions known to contain introns in a substantial number of invertebrate and vertebrate tropomyosin genes (Fig. 6). The simple structural organization of Hydra tropl, which is also evident from Southern analysis (Fig. 5), resembles the simple organization of the tropomyosin gene in yeast (Liu and Bretscher, 1989).

\section{Tropomyosin in tentacle epithelial cells}

As shown by Wood and Novak (1982) and Campbell (1987), nematocytes are anchored in tentacle epithelial cells by a complex configuration of intercellular junctions, termed the nematocyte/battery cell/mesoglea complex. Three structures contribute to this complex: (1) a desmosomal junction between the base of the nematocyte and the upper surface of the epithelial cell. (2) Microtubules and dense cytoskeleton between the upper and lower surfaces of the epithelial cell tongue between the nematocyte and the mesoglea. And (3), an adjacent hemidesmosome with extracellular filaments extending into the underlying mesoglea.

The results presented here show that tropomyosin is present in tentacle epithelial cells at the nematocyte/battery cell/mesoglea junction (Fig. 10 and Fig. 11). The function of this junction is unknown but its location and structure suggest that it is involved in anchoring nematocyte capsules. The finding of tropomyosin at this position suggests that it is a component of the cytoskeleton that forms the nematocyte anchor.

Despite the usual association between tropomyosin and actin in cytoskeleton, we could observe no accumulation of phalloidin-staining material in the junction. This observation agrees with previous results (Wood and Nowak, 1982). However, caution is necessary, since intensely staining myonemes in the same cells could mask phalloidin staining in the junctions. Thus, the exact nature of this tropomyosin-containing structure remains to be elucidated.

\section{Tropomyosin in epithelial cells of the body column}

Western blot and immunocytochemistry indicated a high level of tropomyosin protein in body column cells. Northern analysis, however, showed only a low level of tropl expression in body column cells. Thus, if body column tropomyosin is the product of the tropl gene, it must be a very stable protein. Alternatively, the tropomyosin in body column cells could be the product of a second tropomyosin gene that does not crosshybridize with tropl but whose product is recognized by the chicken antibody. Although at present it is not possible to distinguish between these two possibilities, the existence of a muscle-specific tropomyosin gene in a related coelenterate species (Baader et al., 1993) suggests that there might be additional tropomyosin genes in Hydra.

This study raises several questions. (1) What is the function of the tropomyosin cushion? (2) Are there components other than tropomyosin involved in this structure and how are these components organized? (3) What initiates the formation of the tropomyosin cushion in Hydra battery cells? One attractive hypothesis would be that the epithelial cell-nematocyte interaction triggers the synthesis of tropomyosin. (4) Finally, how is Hydra tropl related to tropomyosins of simple (yeast) and more complex organisms? The answers should contribute to our understanding of both neatocyte function in Hydra and the role of tropomyosin in nonmuscle cells.

We thank Günter Gerisch, Max Planck Institute für Biochemie, Martinsried, for the use of his laser scanning microscope; and Betina Mühlbauer and Richard Albrecht for assistance with confocal mis- 
croscopy. We are also grateful to Gabi Praetzel for expert technical assistence; Günther Plickert and Christine Baader for helpful discussion; Hans Bode and Michael Sarras Jr for providing a Hydra cDNA library; Judith Ittner-Bosch for dog muscle protein; and Anthony Bretscher for a gift of anti-yeast tropomyosin antibody. M.S.L.H. is supported by the Universidad Autonoma de Madrid, L.M.S. received a post-doctoal fellowship from the Alexander-von-Humboldt Stiftung, Bonn. This work was supported by the DFG (grants to C.N.D. and T.C.G.B.)

\section{REFERENCES}

Baader, C. D., Schmid, V. and Schuchert, P. (1993). Characterization of a tropomyosin cDNA from the hydrozoan Podocoryne carnea. FEBS Lett. 328, 63-66.

Bosch, T. C. G., Krylow, S. M., Bode, H. R. and Steele, R. E. (1988) Thermotolerance and synthesis of heat-shock proteins: these responses are present in Hydra attenuata but absent in Hydra oligactis. Proc. Nat. Acad. Sci. USA 85, 7927-7931.

Bosch, T. C. G., Unger, T. F., Fisher, D. A. and Steele, R. E. (1989). Structure and expression of STK, a $s r c$-related gene in the simple metazoan Hydra attenuata. Mol. Cell. Biol. 9, 4141-4151.

Campbell, R. D. (1987). Organization of the nematocyst battery in the tentacle of hydra: arrangement of the complex anchoring junctions between nematocytes, epithelial cells, and basement membrane. Cell Tiss. Res. 249, 647-655.

Gerisch, G., Noegel, A. A. and Schleicher, M. (1991). Genetic alteration of proteins in actin-based motility systems. Annu. Rev. Physiol. 53, 607-628.

González-Agosti, C. and Stidwill, R. (1992). The contributions of microtubules and F-actin to the in vitro migratory mechanisms of Hydra nematocytes as determined by drug interference experiments. Exp. Cell Res. 200, 196-204
Hobmayer, B., Holstein, T. W. and David, C. N. (1991). Tentacle morphogenesis in hydra. II. Formation of a complex between a sensory nerve cell and a battery cell. Development 109, 897-904.

Karlik, C. C. and Freyberg, E. A. (1986). Two Drosophila melanogaster tropomyosin genes: structure and functional aspects. Mol. Cell. Biol. 6, 19651973.

Kozak, M. (1987). An analysis of 5'-noncoding sequences from 699 vertebrate messenger RNAs. Nucl. Acids Res. 15, 8125-8148.

Kurz, E. M., Holstein, T. W., Petri, B. M., Engel, J. and David, C. N. (1991). Mini-collagens in hydra nematocytes. J. Cell Biol. 115, 1159-1169.

Lees-Miller, J. P. and Helfman, D. M. (1991). The molecular basis for tropomyosin isoform diversity. BioEssays 13, 429-437.

Liu, H. and Bretscher, A. (1989). Disruption of the single tropomyosin gene in yeast results in the disappeareance of actin cables from the cytoskeleton. Cell 57, 233-242.

Marcum, B. A., Fuijsawa, T. and Sugiyama, T. (1980). A mutant strain (sf-1) containing temperature-sensitive interstitial cells. In Developmental and Cellular Biology of Coelenterates (ed. P. Tardent and R. Tardent), pp. 429434. Elsevier/North Holland, Amsterdam.

Ruiz-Opazo, N. and Nadal-Ginard B. (1987). $\alpha$-Tropomysin gene organization. J Biol. Chem. 262, 4755-4765.

Sambrook, J., Fritsch, D. M., and Maniatis, T. (1989) Molecular Cloning: a Laboratory Manual, 2nd edition. Cold Spring Harbor Laboratory Press, Cold Spring Harbor, NY.

Smilie, L. B. (1979). Structure and functions of tropomyosins from muscle and non-muscle sources. Trends Biochem. Sci. 4, 151-155.

Wieczorek, D. F., Smith, C. W. J. and Nadal-Ginard, B. (1988) The rat $\alpha$ tropomyosin gene generates a minimum of six different mRNAs coding for striated, smooth, and nonmuscle isoforms by alternative splicing. Mol. Cell. Biol. 8, 679-694.

Wood, R. L. and Novak, P. L. (1982). The anchoring of nematocysts and nematocytes in the tentacles of hydra. J. Ultrastruct. Res. 81, 104-116.

(Received 30 December 1993 - Accepted 4 March 1994) 